\title{
Increasing Potential Interacting Area of Nanomedicine Enhances Its Homotypic Cancer Targeting Efficacy
}

Jingyi Zhu†‡\#, Cansu Sevencan†\#, Mingkang Zhangz\#, Reece Sean Ashley McCoy†, Xianguang Ding†, Jingjie Yez, Jianping Xie†, Katsuhiko Arigađz, Jun Feng $z^{*}$, Boon Huat Bay ${ }^{*}$, David Tai Leong $\dagger^{*}$

† Department of Chemical and Biomolecular Engineering, National University of Singapore, 4 Engineering Drive 4, 117585, Singapore

*E-mail: cheltwd@nus.edu.sg

$\ddagger$ Key Laboratory of Biomaterials of Guangdong Higher Education Institutes, Department of Biomedical Engineering, Jinan University, Guangzhou 510632, China

z Key Laboratory of Biomedical Polymers of Ministry of Education \& Department of Chemistry, Wuhan University, Wuhan 430072, China

*E-mail: fengjun@whu.edu.cn

$\lambda$ WPI-MANA, National Institute for Materials Science (NIMS), 1-1 Namiki, Tsukuba, Ibaraki 305-0044, Japan.

z Department of Advanced Materials Science, Graduate School of Frontier Sciences, The University of Tokyo, 5-1-5 Kashiwanoha, Kashiwa, Chiba 277-8561, Japan

TDepartment of Anatomy, National University of Singapore, 4 Medical Drive, Singapore 117594, Singapore

*E-mail: antbaybh@nus.edu.sg 


\section{Supporting Figures}

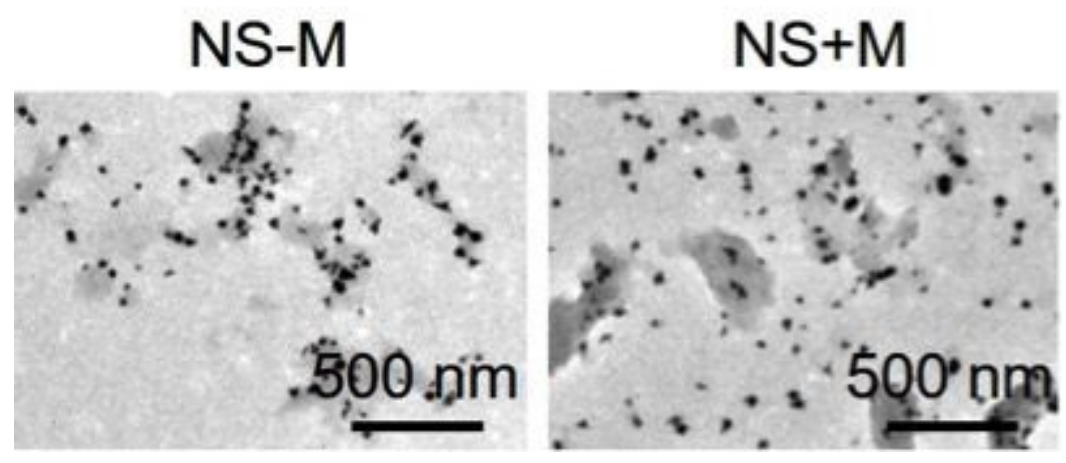

Figure S1. TEM images of in situ synthesis NS-M and NS+M (mixture solution of prior synthesized NS and M). 


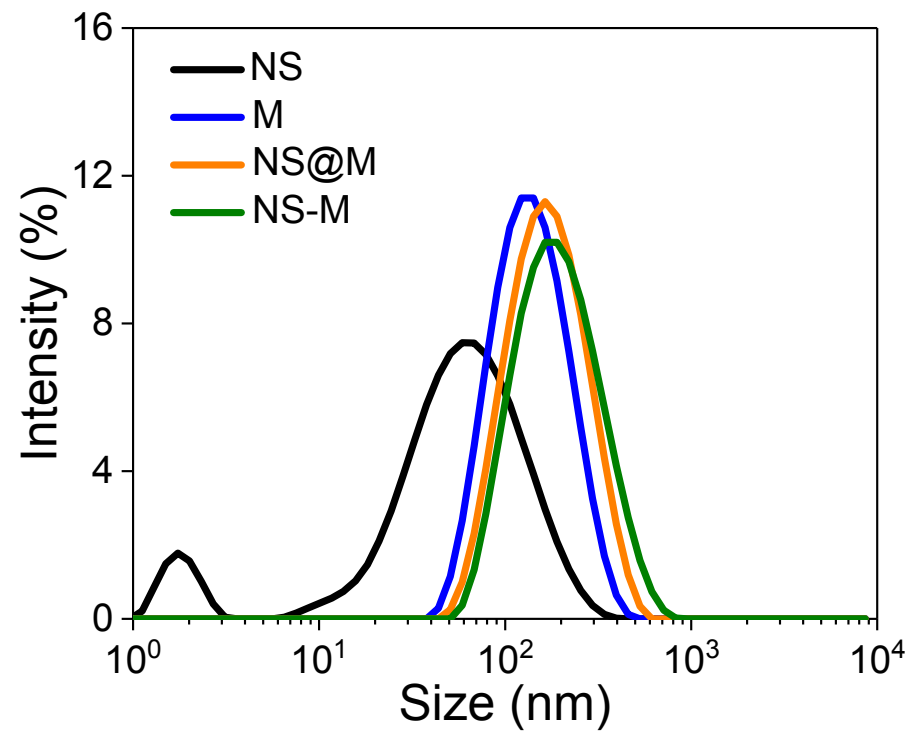

Figure S2. Size intensity curves of NS, NS-M, NS@M, and M measured by DLS. 


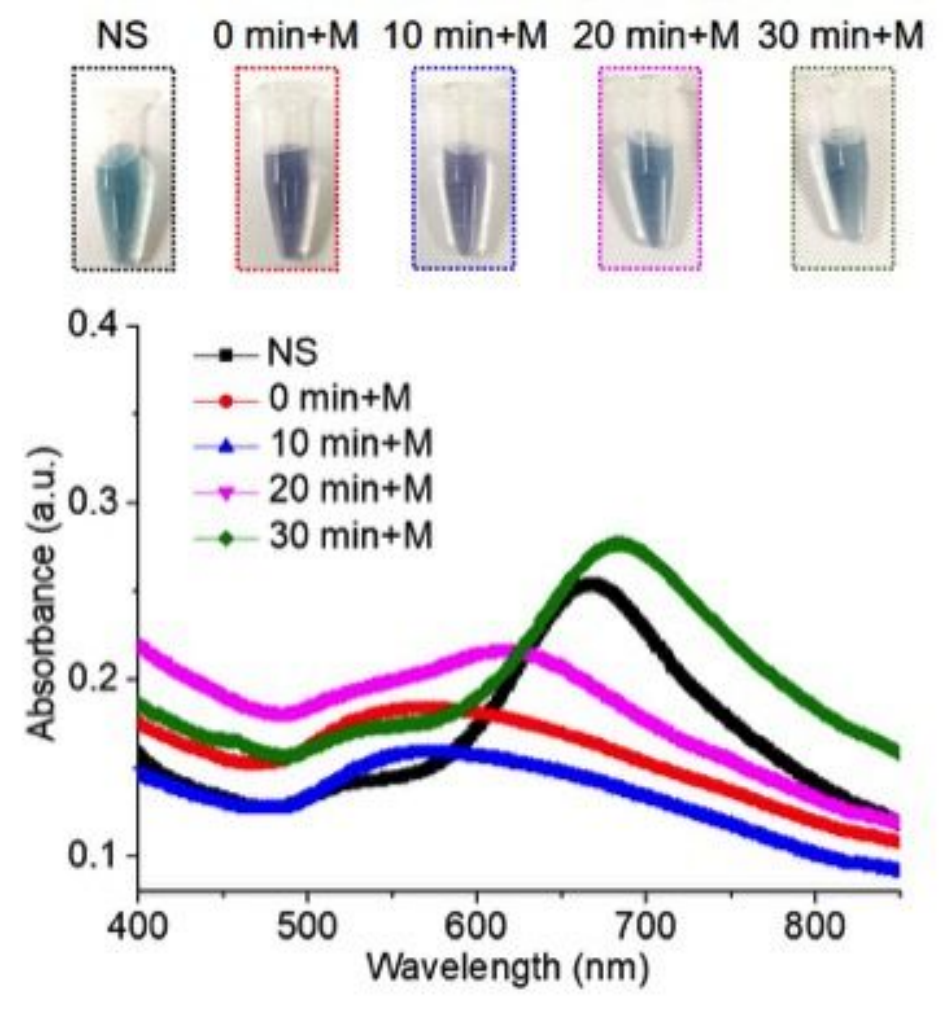

Figure S3. UV-vis absorption spectra of NS and NS-M with the addition of cell membrane materials at $0 \mathrm{~min}, 10 \mathrm{~min}, 20 \mathrm{~min}$, and $30 \mathrm{~min}$. The photos above were results obtained by stirring overnight corresponding to the absorption spectra. 


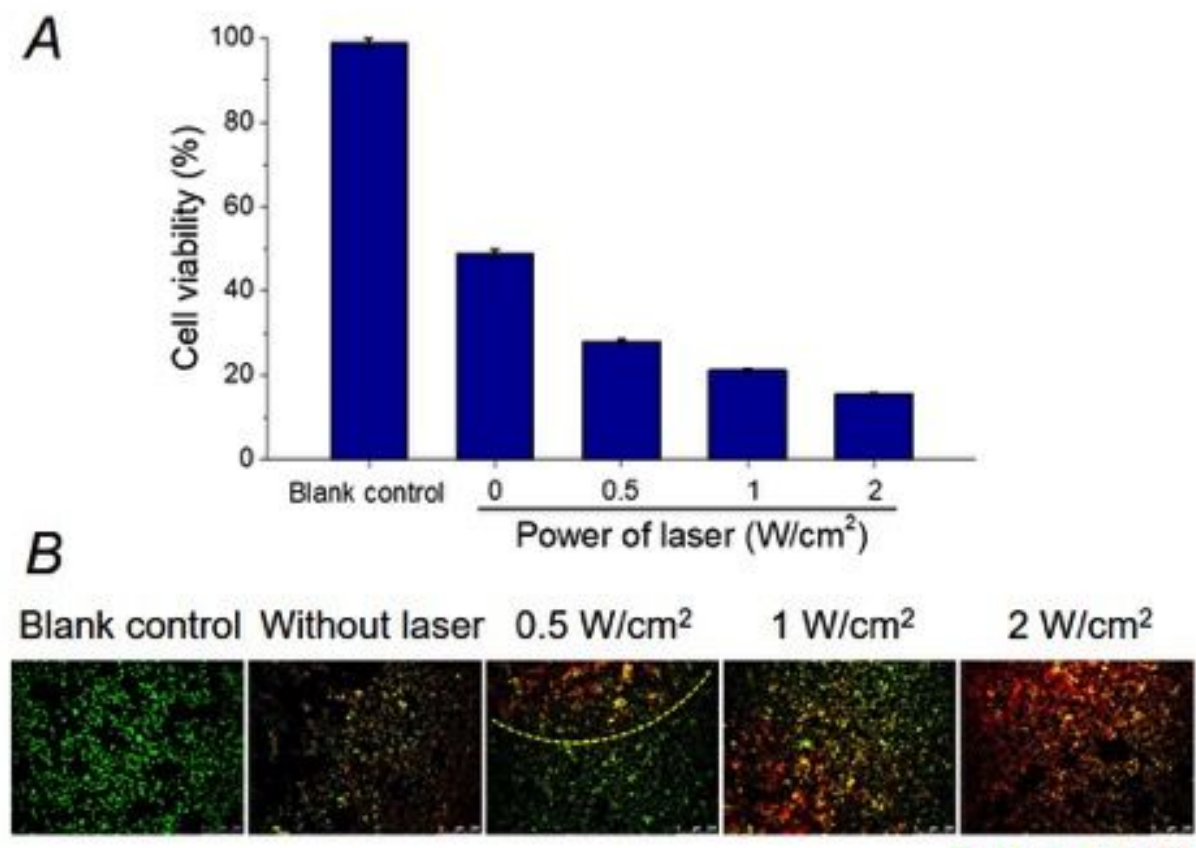

Calcein-AM/PI

Figure S4. (A) In vitro cytotoxicity of NS-M/DOX against B16F10 cells with NIR irradiation for 4 min under different power of laser ranging from 0 to $2 \mathrm{~W} \mathrm{~cm}^{-2}$. Cells without any treatment were served as blank control. (B) The corresponding fluorescence images of B16F10 cells upon various treatment. The live cells were stained green with calcein-AM and dead cells were stained red with $\mathrm{PI}$. 

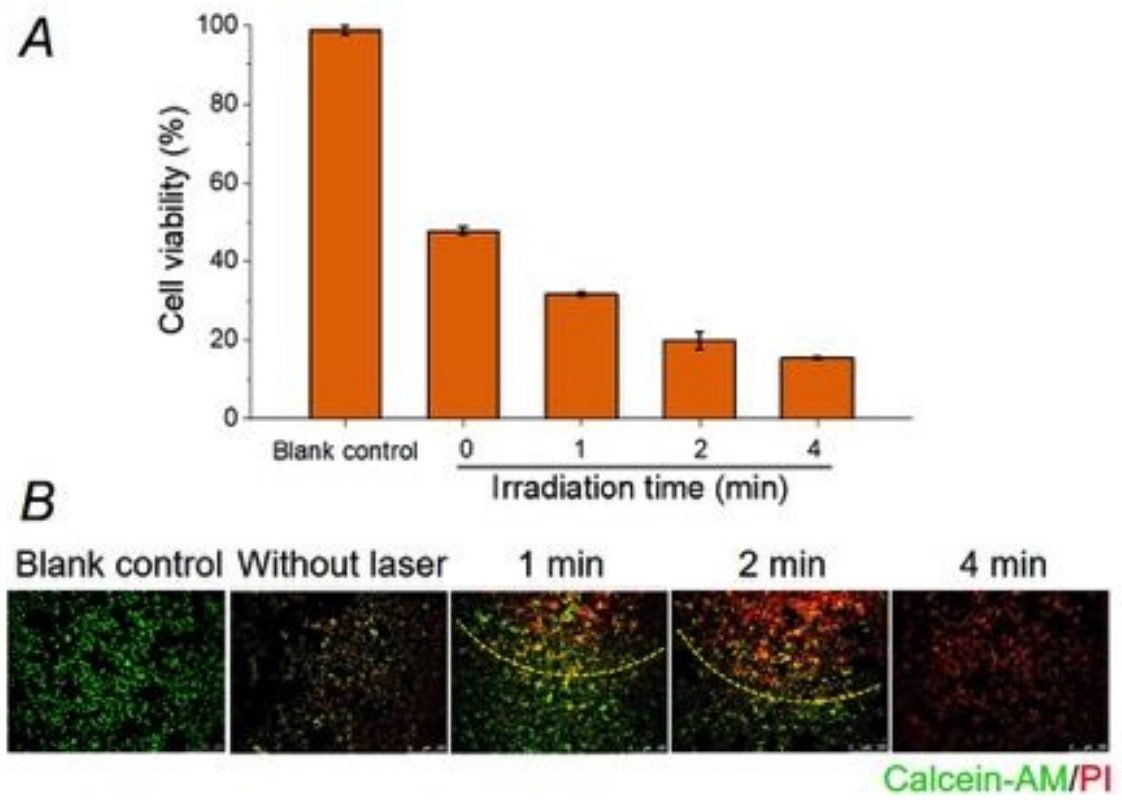

Figure S5. (A) In vitro cytotoxicity of NS-M/DOX against B16F10 cells under a fixed NIR power of $2 \mathrm{~W} \mathrm{~cm}^{-2}$ for different time periods ranging from 0 to $4 \mathrm{~min}$. Cells without any treatment were served as blank control. (B) The corresponding fluorescence images of B16F10 cells upon various treatment. The live cells were stained green with calcein-AM and dead cells were stained red with PI. 